\title{
Application of SPH on Numerical Simulation of Two-Dimensional Steady and Unsteady Flow
}

\author{
Jiang-chuan LIU, Zhi-gang YIN, Wei JI \\ School of Water Resources and Environmental Engineering, Changchun Institute of Technology, Changchun \\ 130012, China
}

\begin{abstract}
Smoothed particle hydrodynamics (SPH) method is a new developed numerical method. The paper studies two-dimensional steady and unsteady flow based on the SPH and considered a variety of conditions. SPH is applied to orifice outflow. The feasibility of model is verified though the comparison between theoretical solution and numerical results. The paper applies the SPH model to the flow of liquid with free surface on Laxiwa dam, and simulates the process of the discharge of the surface outlet. The water recharge boundary is used in the model. The paper gives an analysis between numerical pressure and experimental pressure. The results indicate that it's possible to simulate and research two-dimensional steady and unsteady flow by the smoothed particle hydrodynamics method.
\end{abstract}

KEYWORD: SPH; orifice outflow; surface outlet discharge; water recharge boundary

\section{INTRODUCTION}

The smoothed particle hydrodynamics method, also known as the SPH, is a relatively new numerical method which is developed in the field of computational fluid in recent years. It is originally used to solve the astrophysics group involved in the flow of the physical boundary conditions without any liquidity problems in three-dimensional space [1][2]. The SPH is an absolute element-free method, When calculating the spatial derivatives, the grid is not used in the SPH which is different from the finite element method and finite difference method based on the grid numerical method, but with a series of randomly distributed particles which can move freely according to the laws of mechanics of fluid flow and own all the mechanics quantities to represent the entire continuum fluid, the basic equations of fluid mechanics are transformed into the numerical calculation equation of the SPH through a process known as the kernel functions of function value to approximate integral kernel $[3][4][5]$.

\section{THE THEORETICAL FOUNDATION OF THE $\mathrm{SPH}$}

\subsection{Principle solution of $\mathrm{SPH}$}

The theoretical foundation of the SPH method is derived from the particle method; it is that the continuous quantities are presented by a series of parameters such as particle density and pressure in the collection for interpolation. The equations of fluid mechanics are converted to the models that are required by calculation through evaluating the kernel function of particles (or nodes) [6][7]. Since all mechanical quantity are loaded by the particles, so integral equation is evaluated by the summation of a series of discrete points. In this way, we simply need to solve the ordinary differential equation containing time derivative without solving the partial differential equation containing the time and space derivative at the same time [8].

Any macro variables in the circuit (density 、 pressure v temperature and so on) are presented by the interpolation of a series of disordered points [3]

$$
<A(r)>=\int_{D} A\left(r^{\prime}\right) W\left(r-r^{\prime}, h\right) d r^{\prime}
$$

Where $\mathrm{D}=$ the whole solving area; $\mathrm{A}(\mathrm{r})=$ Macro variable pre-set point value; $\mathrm{h}=$ Smooth Length; $W\left(r-r^{\prime}, h\right)=$ The interpolation kernel, It has two independent variables, that is The distance between the particles $\left|r-r^{\prime}\right|$ and the Smooth Length $h$.

Mass conservation equation

$$
\frac{d \rho_{i}}{d t}=\sum_{j=1}^{N} m_{j}\left(\boldsymbol{u}_{i}-\boldsymbol{u}_{j}\right) \cdot \nabla W\left(r_{i}-r_{j}, h\right)
$$

Momentum conservation equation

$$
\frac{d \boldsymbol{u}_{i}}{d t}=-\sum_{j=1}^{N} m_{j}\left(\frac{P_{i}}{\rho_{i}^{2}}+\frac{P_{j}}{\rho_{j}^{2}}\right) \nabla W\left(r_{i}-r_{j}, h\right)
$$


Energy conservation equation

$$
\frac{d e_{i}}{d t}=\frac{P_{i}}{\rho_{i}^{2}} \sum_{j=1}^{N} m_{j}\left(\boldsymbol{u}_{i}-\boldsymbol{u}_{j}\right) \cdot \nabla W\left(r_{i}-r_{j}, h\right)
$$

Particle motion position

$$
\frac{d \boldsymbol{r}}{d t}=\hat{\boldsymbol{u}}_{i}=\boldsymbol{u}_{i}+\varepsilon \sum_{j=1}^{N} m_{j} \frac{\boldsymbol{u}_{j}-\boldsymbol{u}_{i}}{\bar{\rho}_{i j}} W\left(r_{i}-r_{j}, h\right)
$$

\subsection{Boundary condition processing}

The particle density is calculated by summing the density value of the particles around in the SPH model, because there are no fluids particles beside the free surface, the calculation of the free surface particle density must be less than the actual value, and the pressure is a small amount which is not in conformity with the actual value, all those factors can affect the calculation of numerical stability and accuracy. It will be considered as free surface particles if the density of particles satisfies the following conditions, forcing its density is equal to the free surface of fluid density[7].

$$
\rho_{i}<\beta \rho_{0}
$$

The method of boundary force is often used for the processing of solid side wall. In the two dimension, the domain of the force affect is particle spacing circle domain; in the three dimension, the domain of the force affect is particle spacing of the ball field. The form of the force is the form of the intermolecular interaction. For example, when processing the boundary particles and forces of fluid particles, the body force of unit mass can be the form of Lennard-Jones force:

$$
f(\boldsymbol{r})=\frac{D\left[\left(\frac{r_{0}}{r}\right)^{p_{1}}-\left(\frac{r_{0}}{r}\right)^{p_{2}}\right] \boldsymbol{r}}{r^{2}}
$$

\section{ENGINEERING EXAMPLE}

\subsection{The orifice outflow}

Using the SPH method for the numerical simulation of unsteady flow problems of orifice, various parameters of the mathematical model are as Fig.1 and Fig. 2 .

The calculation model of Container is shown in figure 1, The container size: $0.2 \mathrm{~m} \times 0.25 \mathrm{~m}$; the Size of the liquid: $0.2 \mathrm{~m} \times 0.2 \mathrm{~m}$.

The initial distance of the particles $\mathrm{d} 0$ is amount to spatial step length of grid method. If the particle size is larger, the oscillation is significant, if the particle size is too small, the number of particles will be increased, and the calculation process will take more time. According to the actual calculation conditions, $\mathrm{d} 0=0.0025 \mathrm{~m}$.
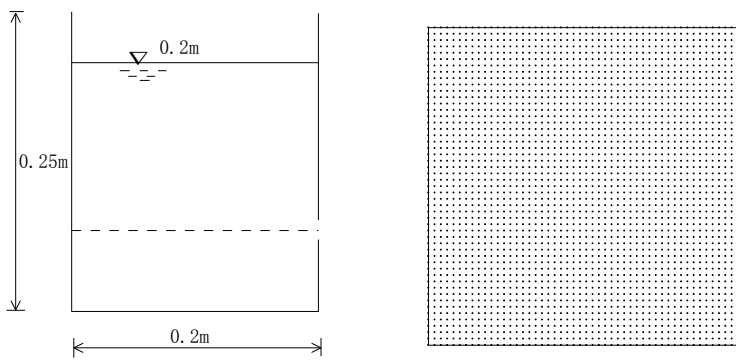

Fig.1 Numerical experiment model Fig.2 Disposal of initial particles

The whole calculation domain is discretized according to the container and the particle size, As shown in figure 2 the number of Fluid particles are 5200 , the number of The boundary particle 286.

The numerical calculation of the SPH flow field at each time is shown as Fig 3.
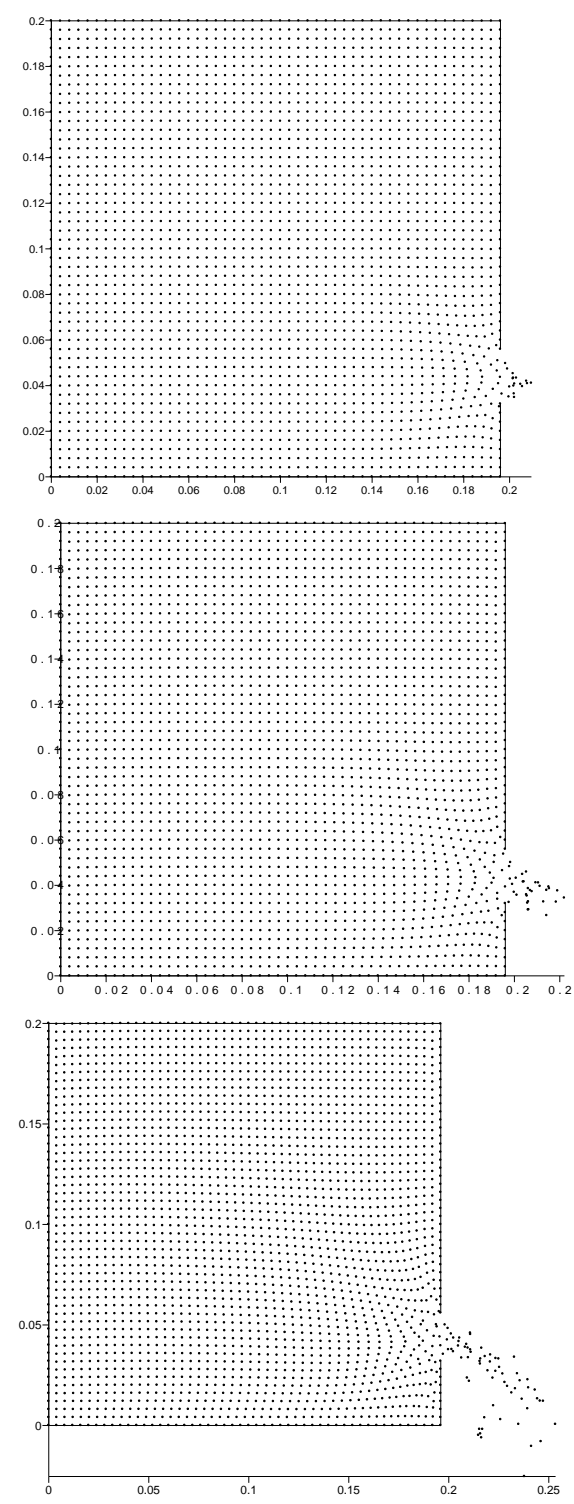

Fig. 3 Calculating distribution of particles at $0.03 \mathrm{~s}$ to $0.1 \mathrm{~s}$

With the change of time, The flow outflows continuously from the hole, because the SPH tracks the particle itself in the calculation process, the particles disappearing from the container with the time increasing. As can be seen from the calculations, the flow field of each time and particle 
distribution are in accord with the actual orifice flow. Variation curve of water level with time changing as shown in figure 3,there's some slight deviation between the calculation value and the theoretical value, which is caused by the fluctuation of the free surface in numerical calculation process, but for the whole process of flow, the SPH numerical results and the actual results of theoretical calculation are basically coincided.

\subsection{Surface outlet discharge}

In the surface hole discharge of Rasiva hydropower, due to constant upstream of the upper reservoir, the whole process of flow is a steady state, according to the actual situation and physical experiment, in the SPH mathematical model, the method of water recharge boundary to meet the condition of steady state flow, numerical experiment parameters are as fig 4 and fig5.

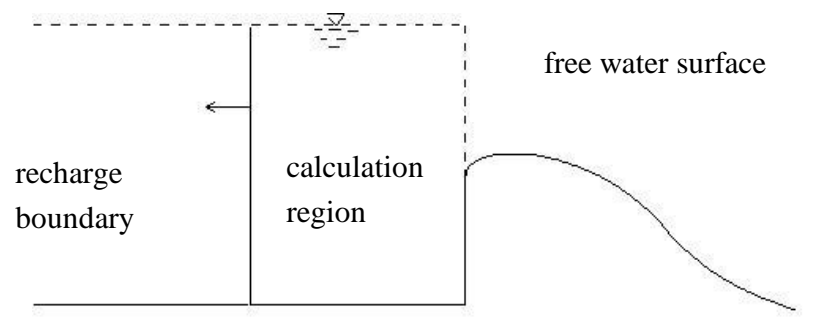

Fig.4 Numerical experiment model

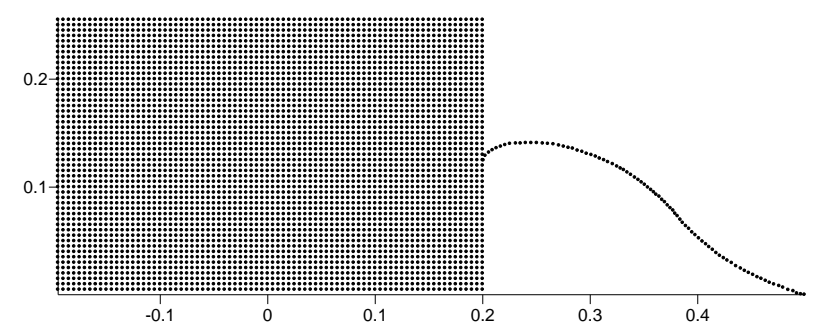

Fig.5 Disposal of initial particles

The particles move along with the change of time. The water flow move from the initial time, with the increasing of time, the flow field will change until stable. (Such as Fig 6). Pressure of measuring point of numerical experiment and physical experiment as Fig 7.

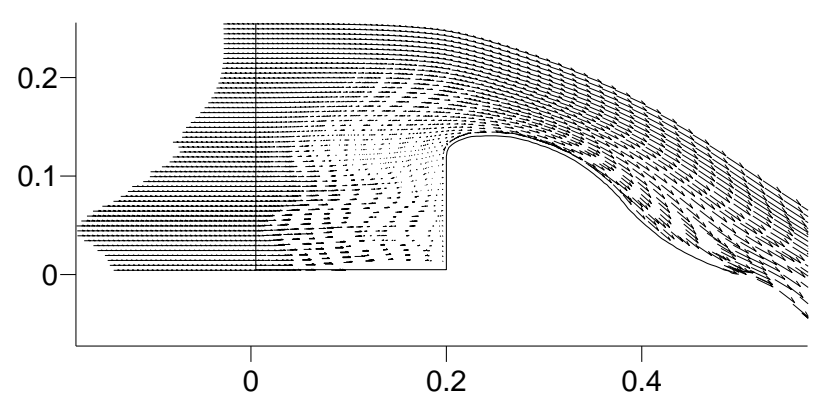

Fig.6 Calculating flow field at $0.4 \mathrm{~s}$
It can be seen from the numerical experiments of flow field and particle distribution, the water tongue, flow pattern and other results is consistent with the actual situation. According to verify the measuring point of pressure, the pressure distribution trends of the measuring point is consistent with each other, but there are some deviations with the measured values, the analysis of the reason may be: the method of numerical calculation itself is that taking a finite number of discrete points; The actual surface hole should include the following several parts: import segment, straight segment, WES curve segment. The surface hole boundary, generated by the numerical experiment, having some deviations in the above several parts, which will inevitably influence the accuracy of the numerical results.

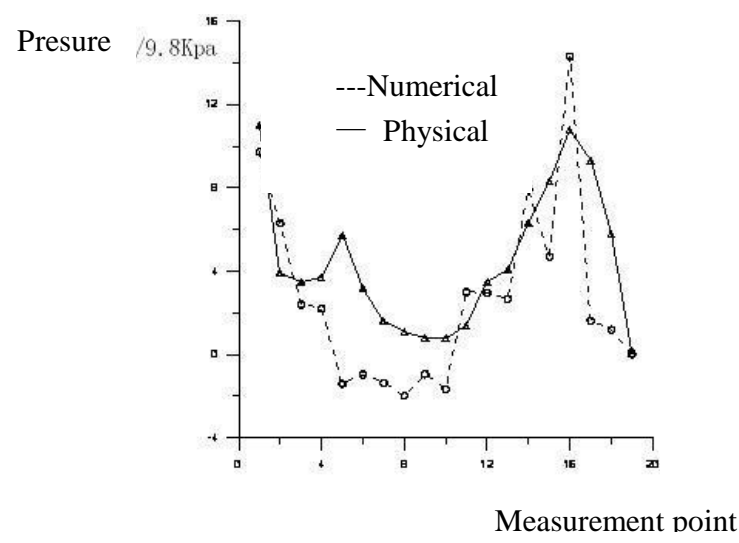

Fig.7 Pressure comparison

\subsection{The Conclusion}

Traditional numerical methods are based on grid models, but both the Euler grids and Lagrangian meshes need to establish fixed topological relationship between the grids, which limits the practical application of grid method. Using the SPH method, a mathematical model of the dam discharge surface hole is established in this paper, verified by the physical model, and suggest that the smooth particles can be used to solve nonlinear large deformation in high speed water flow.

Due to the effect of upstream reservoir, the discharge process is a steady state condition. So the Hydrating boundary is put forward to meet the steady state flow. That provides a model for the SPH simulation of the flow calculation problem.

\section{ACKNOWLEDGEMENTS}

This work was financially supported by the Project of Jilin Province Department of Education(Grant No. 2014312), and the Youth Fund of Changchun Institute of Technology(Grant No.320130030). 


\section{REFERENCES}

[1] Lucy L B, A Numerical Approach to The Testing of The Fission Hypothesis, The Astronomical Journal, 82. 10131024 (1977)

[2] Monaghan J J, Smoothed Particle Hydrodynamics, Annual Review of Astronomy and Astrophysics, 30. 543-574 (1992)

[3] Zhang Suo-chun, Smoothed Particle Hydrodynamics (SPH), Chinese Journal of Computational Physics, 4. 385397 (1996) (In Chinese)

[4] LI Da-ming, LIU Jiang-chuan, etal, Application of SPH to Numerical Simulation of Discharge through Crest Orifice on Dam, Advances in Water Science, 19(6). 841-845 (2008) (In Chinese)
[5] YANG Xiu-feng, PENG Shi-liu, Simulation of Dam-break Flow with SPH Method, Chinese Journal of Computational Physics, 27(2). 173-180 (2010) (In Chinese)

[6] SUN Fang-jin, ZHANG Da-ming, Study on Fluid-Structure Interaction based on Smoothed Particle Hydrodynamics, J. Earthquake Eng. And Eng. Vibration, 33(6). 222-227 (2013) (In Chinese)

[7] HAN Ya-wei, QIANG Hong-fu, ZHAO Jiu-ling, etal, A New Repulsive Model for Solid Boundary Condition in SPH, Acta Phys. Sin. 62(4). 044702(1-10)(2013)(In Chinese)

[8] LI Da-ming, WANG Zhi-chao, BAI Lin, etal, Investigations on the Process of Droplet Impact on an Orifice Plate, Acta Phys. Sin. 62(19). 194704(1-11) (2013) (In Chinese) 